\title{
A Novel Recognition Model of University Students' Psychological Crisis Based on DM
}

\author{
Lihuang Cai $\mathbb{D}$ \\ School XiaMen Institute Of Technology, Xiamen 361021, China \\ Correspondence should be addressed to Lihuang Cai; cailihuang@xit.edu.cn
}

Received 30 December 2021; Revised 17 January 2022; Accepted 18 January 2022; Published 10 February 2022

Academic Editor: Tongguang Ni

Copyright $(92022$ Lihuang Cai. This is an open access article distributed under the Creative Commons Attribution License, which permits unrestricted use, distribution, and reproduction in any medium, provided the original work is properly cited.

\begin{abstract}
The research on identifying college students' psychological crises can assist college administrators in better understanding of students' psychological changes and planning of intervention measures ahead of time to ensure college students' mental health. When confronted with unexpected events or major setbacks and difficulties, people experience psychological crises, which they can neither avoid nor solve using their own resources and stress methods. This paper proposes a method of college students' psychological crisis identification based on data mining technology to solve the problems that exist in the process of identifying psychological crises in college students and to improve the correct rate of identifying psychological crises in college students. In comparison to other technologies, data mining can uncover the link between students' psychological problems and their basic information, as well as the main causes of psychological problems, using statistical data.
\end{abstract}

\section{Introduction}

Psychological crisis refers to the serious psychological imbalance that individuals can neither avoid nor use their own resources and stress methods to solve when encountering unexpected events or major setbacks and difficulties [1]. Because of the differences in cultural background, family background, educational experience, individual development period, and personal experience, the situation of psychological crisis is not absolute. At the same time, each person's individual crisis varies with the change of time, place, event, and situation, and university students' psychological crisis identification has its own characteristics. When Hopfield fails to meet the objective function's requirements, the penalty function structure can be used to control the objective function of identifying psychological crises among university students, greatly reducing error rates and making network maintenance easier. As a result, current research is concentrating on the identification of university students' PCS. In the field of mental health education, incorporating big data technology into the identification of students' psychological crises, collecting and analyzing data from university students' daily lives at school, and improving the timeliness and accuracy of psychological crisis identification have become a new topic for psychological crisis identification workers in the information age. Every crisis has its particularity, and every crisis is unique and different $[2,3]$. When we grasp and analyze the overall situation and start with the nature of the psychological crisis, we can still divide the crisis into three categories: first, developmental crisis, which is also called adaptive crisis or maturity crisis. It refers to the abnormal stress reaction caused by the sudden change of environment or self-physiology in the process of individual growth and development. Second, the situational crisis [4] refers to a situation in which individuals are unable to predict or control rare or unexpected extraordinary events. The key difference between a situational crisis and other crises is that the extraordinary events that trigger the crisis are unpredictable or difficult for university students to control, such as a parent's divorce, violent aggression, or strong interpersonal conflicts [5]. At this time, domestic research on PCS 
identification has been thoroughly examined from early warning to prevention to intervention, yielding some promising findings. However, most of the studies focus on the explicit crisis, such as what kind of external performance the PCS identification will have, with less attention paid to the implicit crisis [6].

In order to solve the problems existing in the process of university students' psychological crisis identification and improve the correct rate of university students' psychological crisis identification, this paper proposes a method of university students' psychological crisis identification based on DM technology. DM is a technology of data management, analysis, and processing, and a key step of knowledge discovery. The main applications of this method are correlation analysis, classification [7, 8], clustering, prediction time series model [9], and deviation analysis. However, the system designed by the system dynamics method is subjective in data classification, and the causal relationship is obscure and difficult to explain. The mental health analysis system designed by other weighted fuzzy logic theories [10] only stays at the data operation and management level such as data acquisition, statistics, query, and report, and the utilization efficiency of data is not high. The basic process of DM, a large number of DM, according to the definition of management, the preprocessed data get a reliable format, which is convenient for the mining wizard to process the data, and then enter the mining kernel to get the pattern set, which can express and explain the comprehensive knowledge base that users can understand and use after the pattern filtering of the mining manager.

The basic process of DM is as follows: a large number of raw data are extracted after data preprocessing according to the mining manager's data definition to obtain data in a correct and reliable format that is easy to process by the mining wizard, and then entered the mining kernel to obtain a pattern set that is expressed and explained, and then filtered by the mining manager to obtain a knowledge base that is easy for users to understand and generalize. They can organize the structure of a neural network according to the specific problems of university students' PCS identification, and they can effectively fit the changing characteristics of university students' PCS [11, 12]. Despite the fact that prevention, early warning, and intervention mechanisms for the psychological crisis have been established, extreme crisis events that appear to occur "for no apparent reason" continue to occur frequently. Because some crises are complex, difficult to identify, and difficult to detect, they can only have serious consequences if they are not addressed quickly and effectively $[13,14]$. This paper examines the unique challenges of identifying psychological crises in university students and collects signals of psychological crisis detection in university students using specialized equipment [15].

\section{Related Work}

According to literature [16], university students' psychological crisis is universal, with complex symptoms and no quick fix, and it is the driving force for university students' growth and change, including the duality of danger and opportunity, as well as having time-specific characteristics. According to literature [17], using the big data analysis method and the ID decision tree algorithm, a data classification mining system model of university students' psychological problems was created. The system mined association rules using the Apriori algorithm. The main factors affecting psychological problems and their relationships were discovered through data testing. According to literature [18] and research, school, family, and societal losses have resulted from students' vicious wounding or selfinjury incidents caused by the psychological crisis. As a result, establishing an effective early warning system for psychological crises among university students is essential for effectively preventing crisis events. According to literature [19], university students' psychological crises are characterized by unexpected events, relief from helplessness, pain, and potentially dangerous consequences. Literature [20] proposes a psychological classifier model based on artificial neural networks and fuzzy mathematics theory and uses training samples to build the model using the MATLAB neural network toolbox, resulting in more accurate classification results. According to literature [21], research shows that differences in the characteristics, forms, and stressors of university students' psychological crises categorize them into different types. The network structure of a mental health prediction system model based on the BP neural network algorithm was established using the artificial neural network toolbox in MATLAB, according to literature [22], in order to predict the mental health of university students. According to literature [23], through the method of big data analysis, the psychological crisis of university students is mainly attributed to four aspects: the crisis caused by physiological and psychological contradictions; crisis caused by interpersonal tension; psychological crisis caused by the market economy and employment pressure; crisis caused by sexual physiology maturity and sexual psychology imperfection. According to literature [24], research shows that the diagnosis experience of domestic and foreign psychologists is introduced into the expert system of mental disorder diagnosis through the expert system of the artificial neural network, so that the system can detect and diagnose students' mental health in all aspects, which provides convenience for improving the function of mental health education for university students. Literature [25] puts forward that the BP neural network algorithm is applied to university students' psychological disorder identification, and combined with "artificial intelligence" to establish a model, which can be used by university students to realize online self-diagnosis of psychological disorder categories, thus reducing the workload of some mental health managers and reducing the working pressure of college psychological counseling institutions.

Based on DM, this paper studies the identification of university students' PCS, and some achievements have been made in the application research of DM technology in psychological crisis prevention. The feasibility and effectiveness of DM technology in analyzing and predicting students' mental state, evaluating students' mental health, pattern recognition of psychological diagnosis, and early 
warning of psychological crisis have been proved. The application of DM in the classification technology model, psychological obstacle identification model, and psychological condition prediction network model of psychological crisis prevention has also achieved relevant research results.

\section{Principle and Basic Steps of DM Technology}

3.1. Principle and Algorithm of DM. DM is a process of extracting potential and useful information and knowledge from a large number of incomplete, fuzzy, noisy, and random data. The extracted knowledge is expressed as rules, concepts, laws, patterns, and so on. In the process of identifying university students' PCS, it is a crucial step to collect university students' PCS signals first. At present, sensors are mainly used to collect university students' PCS signals. The decomposition scale of wavelet transform is determined, and multiscale decomposed the original university students' PCS identification signal to separate the noise from the useful university students' PCS identification signal, which will correspond to different wavelet coefficients. Therefore, this paper uses laser sensors to collect students' PCS signals, which can reduce the cost of collecting PCS signals. The basic process of DM is as follows: a large number of raw data are extracted after data preprocessing according to the mining manager's data definition to obtain data in a correct and reliable format that is easy to process by the mining wizard and then the mining kernel is entered to obtain a pattern set that is expressed and explained and then filtered by the mining manager to obtain a knowledge base that is easy to understand and generalize. The DM model is shown in Figure 1.

DM is an instance-based inductive classification algorithm. For a given data set, this algorithm can create a decision tree model and extract intuitive and simple knowledge of classification rules. To begin, each of the classification measure data set's attribute values is classified and chooses the root node. Take, for example, a university's psychological management system. Some problems that are relatively unseen can only be revealed in a high-stress environment. There will be one-sided problems in the process of evaluating an individual's psychological state if we cannot realize dynamic data management and track and record the data of students at school. This traditional static data management method clearly lags behind students' psychological change processes, reducing the precision and timeliness of psychological crisis intervention. There are four modules in the current psychological management system: student/teacher service, psychological counselor module, parent module, and administrator module. To obtain the target DM set, raw data must be processed. Based on the above mining algorithms, the structure of the psychological crisis system based on three DM methods is shown in Figure 2.
Let the original university students' PCS signal.

$$
y(n)=s(n)+n(n),
$$

where $s(n)$ represents a useful signal of PCS of the university student.

Let $\psi(t) \in \mathrm{L} 2(\mathrm{R})$, whose Fourier transform is $\psi^{\prime}(\omega)$, and the mother wavelet of wavelet transform is defined as

$$
C_{\psi}=\frac{\int_{R}\left|\psi^{\prime}(\omega)\right|^{2}}{|\omega| d \omega<\infty} .
$$

$\psi(t)$ is expanded and translated to obtain

$$
\psi_{a, b}(t)=\frac{1}{\sqrt{|a| \psi(t-b / a)}} .
$$

The calculation formula of the wavelet coefficient is

$$
\omega_{j, k}=\int_{-\infty}^{+\infty} y(n) \psi_{a, b}(n) \mathrm{d} t=\left\langle f, \psi_{a, b}\right\rangle .
$$

The hard threshold method can be described as

$$
T_{h}\left(\omega_{j, k}, \delta\right)=\left\{\begin{array}{l}
\omega_{j, k}, \quad\left|\omega_{j, k}\right| \geq \delta \\
0, \quad\left|\omega_{j, k}\right|<\delta
\end{array} .\right.
$$

The function $\varphi(x k)$ introduces to process the signal data $x k$ of university students' psychological crisis so that it can meet the following conditions:

$$
\sum_{k=1}^{N} \Phi\left(x_{k}\right)=0
$$

The covariance matrix $c \varphi$ of the training data set of university students' PCS is calculated as follows:

$$
C_{\Phi}=\frac{1}{N} \sum_{i=1}^{N} \Phi\left(x_{i}\right) \Phi^{T}\left(x_{i}\right),
$$

and

$$
\lambda_{v}=C_{\Phi} v
$$

3.2. Basic Steps of DM. The implementation steps of DM vary with different application environments, and there are differences for different problems and requirements. Each subtechnology of DM also has its own applicable characteristics and application steps. Complete steps of DM are as follows:

(1) Understand the data and data sources

(2) Obtain relevant knowledge and technology

(3) Integrate and check data

(4) Remove erroneous or inconsistent data

(5) Establish models and assumptions 


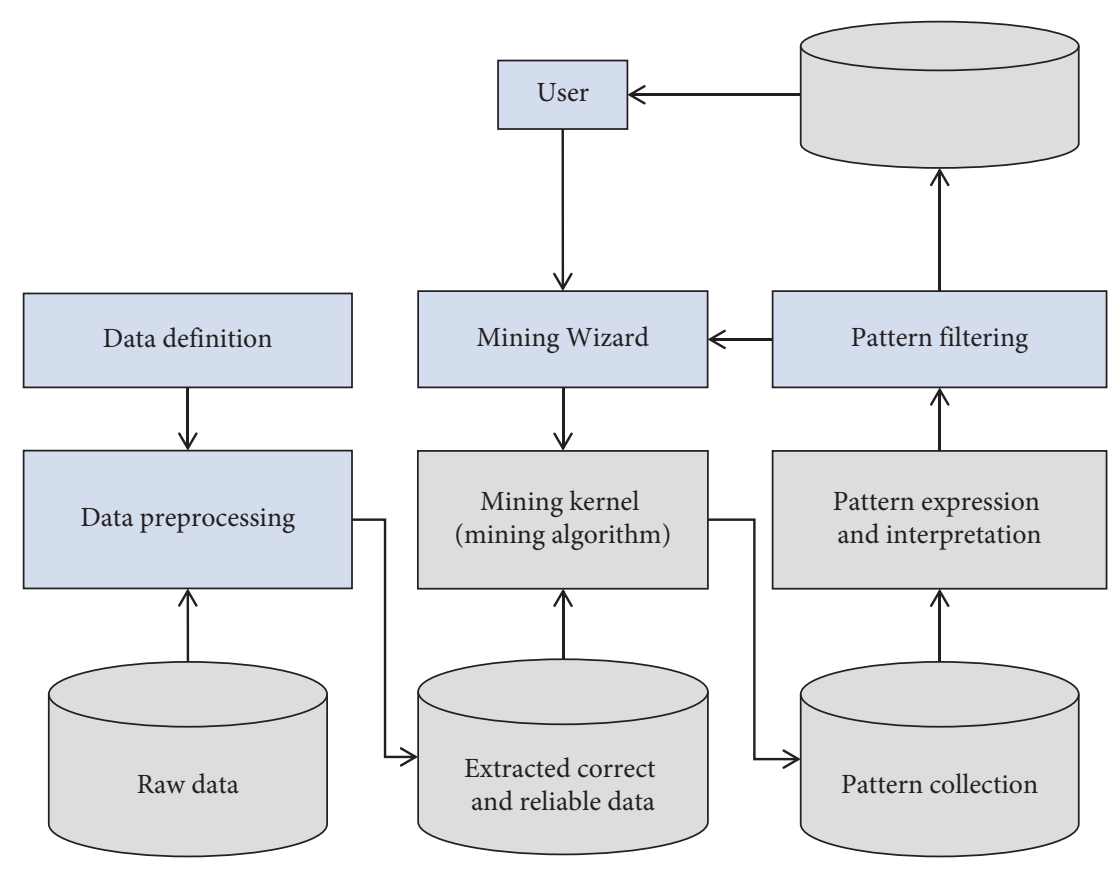

Figure 1: A model of DM.

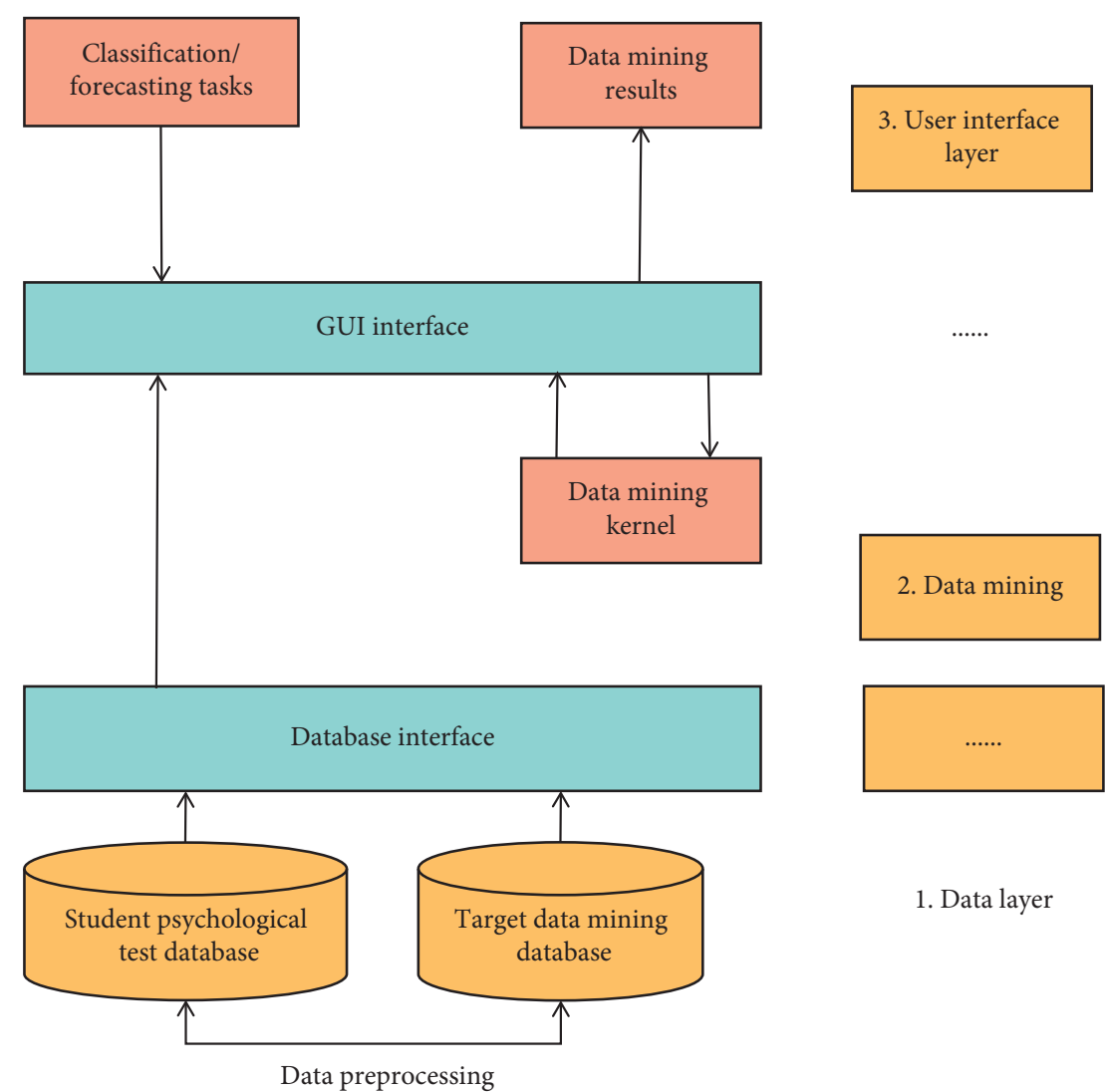

FiguRE 2: Structure diagram of the psychological crisis prevention system based on several DM algorithms.

(6) Actual DM work

(7) Test and verify the mining results

(8) Interpretation and application
This paper examines the specific problems of identifying university students' psychological crises and collects the signals of identifying university students' psychological 
crises using specialized equipment. The wavelet transform's decomposition scale is determined, and then, multiscale decomposed the original university students' PCS identification signal to separate the noise from the useful university students' PCS identification signal, which will correspond to different wavelet coefficients. The performance of the identification model of university students' PCS is verified using test samples, and the identification results of university students' PCS are output. To summarize, Figure 3 depicts the process of identifying university students' PCS based on DM.

The single support vector machine (SVM) method for identifying university students' PCS directly extracts features from university students' PCS without denoising. This method is called SVM for short. The advantages of using neural network algorithm to optimize computer network connection are obvious. First, it effectively improves the connection efficiency of the computer network and makes the network run faster. Second, it effectively reduces the problem of increasing capital investment caused by optimizing computer network connection and maximizes the efficiency of capital utilization; that is, it can get the best optimization effect with the least capital. Traditional intervention did not fully tap the hidden value of student behavior data, which led to the related information being in a state of deep sleep. Only through big data mining and scientific utilization, and effective integration of unrelated and scattered data, can the accuracy of intervention be fully improved. After the analysis of big data students' psychological problems is completed, only by giving feedback to counselors and other psychological staff can the data value be further developed. Technologies such as economics, statistics, computer science, and applied mathematics are commonly used to make use of big data resources. However, psychological workers and managers rarely have such majors, which means that technical personnel are unsure how to intervene in a crisis, and psychological educators are unaware of big data technology's application methods. Students' psychological counseling, Internet access, and loan information, on the other hand, will be subject to privacy protection. More attention from the relevant personnel is required to determine whether this type of data is leaked or not, and whether it can be directly used, among other things.

\section{Research and Implementation of University Students' PCS Identification}

4.1. Identification of University Students' PCS Based on DM. The DM model based on the comprehensive CART decision tree, BP artificial neural network, and pattern recognition, which learn from each other's strengths, is embedded into the current school psychological management system to realize the psychological crisis prevention system. The complex averaging operation is transformed into functional regular operation, which greatly saves the calculation time of university students' PCS identification and realizes better interaction of network information data. Any behavior has a precursor. At present, students' psychological information processing ability is poor, and the sources are relatively few.
Therefore, there is a lack of use-value, which leads to the lack of validity of the data held by counselors and affects their ability of judgment and analysis. Big data have the function of data mining, which can deeply mine and analyze massive data, and effectively predict the development law of things based on it, thus improving the prediction accuracy. Big data analysis can find the rules behind a large amount of data by studying students' external behavior and judge and predict students' behavior patterns and psychological state from all aspects of study and life. To integrate all kinds of data from various channels, it is necessary for the school work leading group to take the lead in establishing a data collection platform for psychological crisis intervention. The collection platform integrates and analyzes the data of various departments, timely provides valuable information for school psychological intervention workers, counselors, class teachers, and other student workers, and improves the predictability, accuracy, and effectiveness of psychological crisis intervention. RBF neural network is a method to identify the PCS of university students. Wavelet analysis is used to denoise the PCS signal of university students, and the characteristics of PCS of university students are extracted from the denoised signal, but RBF neural network is used as the modeling method. The teacher service module provides four submodules of student basic information data management, student measurement management, measurement data management, and other management. Therefore, we can introduce big data technology into the intervention process of university students' psychological crisis. By collecting and processing information such as academic performance, course selection, living conditions, family conditions, interpersonal relationships, and network activity, we can dig and analyze students' psychological state, so as to improve the predictability of students' psychological crisis and give targeted solutions.

As an example, the university's psychological management system is considered. Student/teacher service module, psychological counselor module, parent module, and administrator module are the four modules of the existing psychological management system. Student basic information data management, student measurement management, measurement data management, and other management are the four submodules of the teacher service module. Teachers can filter, query, sort, modify, print, and delete data in the measurement results management module. Researchers collect data on university students' mental health status using clinical scales, psychological investigations, daily outpatient services, and other methods, but the results are rarely shared with counselors, class teachers, and other student workers in the traditional mode of psychological crisis. Furthermore, aside from the aforementioned methods of obtaining psychological data, psychological workers may find it difficult to obtain data from other departments, or they may be unable to mine and process data efficiently. Some students will beautify and cover up problems from a quality standpoint. Furthermore, the information gathered in this manner is primarily stored in a static format, making it difficult to track students' development effectively. Furthermore, college students' psychology is insufficiently 


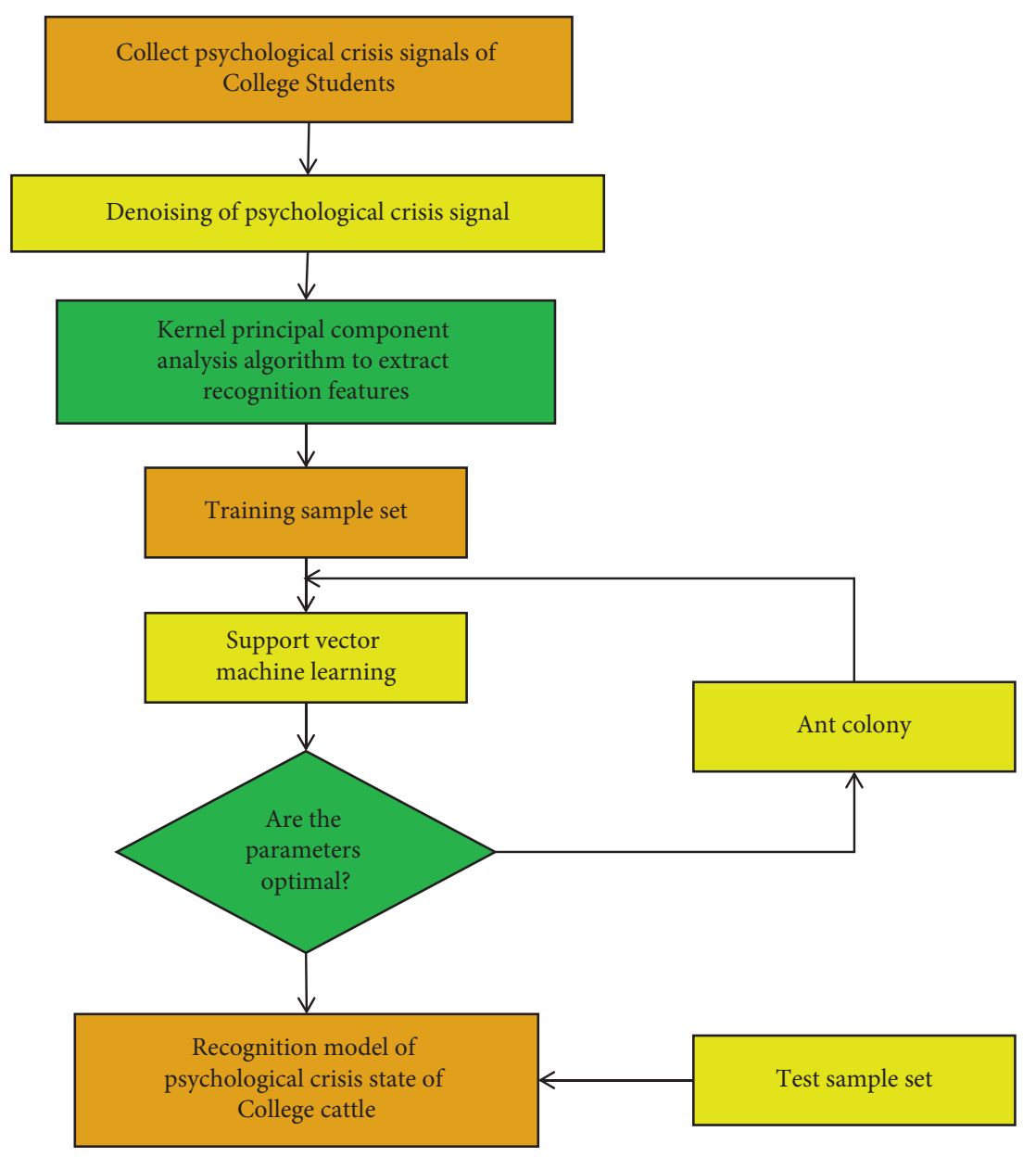

FIgUre 3: Process of identifying university students' PCS by DM.

mature. The psychological state will fluctuate as a result of unexpected events and environmental changes in a complicated social environment. The complex averaging operation is transformed into a functional regular operation, greatly reducing the calculation time for university students' PCS identification and allowing for better network information data interaction. Finally, a model is added to the neural network algorithm to fill in the gaps. When Hopfield fails to meet the objective function's requirements, the penalty function structure can be used to control the objective function of identifying psychological crises among university students, lowering the error rate, and making network maintenance easier. Furthermore, aside from the aforementioned methods of obtaining psychological data, psychological workers may find it difficult to obtain data from other departments, or they may be unable to mine and process data efficiently.

In the process of university students' growth, we should always give them the correct education. In the process of crisis, we should provide more help and attention to university students, provide multifaceted and multiangle social support, and guide university students to actively deal with a hidden psychological crisis. The data of students in school are distributed in various departments of the school, such as the number of students failing courses, elective courses, and punishment in the Academic Affairs Office. In the student office, students participate in social practice and competitions. Psychological investigation, consultation room, and clinical scale are all available at the psychological center. Tuition payments and student loans are handled by the finance department. Students' access to dorms, campus network usage, and other logistics are all part of the logistics package. An effective educational countermeasure system is conducive to effectively preventing university students' hidden psychological crises, timely detecting and intervening, assisting university students in smoothly navigating the crisis, enhancing their ability to resist the crisis, while also being conducive to family and school stability and the harmonious development of society. The university students' psychological crisis status is determined using the school's student psychological management system, and the information is stored in the psychological census database. By analyzing the psychological test data of university students' psychological crisis status identification in the 
screening data, which stores the original data of student information, psychological work evaluates students' psychological status.

4.2. Experimental Results and Analysis. In order to reflect the fairness of the experimental results of university students' PCS identification and increase the persuasion of the results, three experiments were carried out, respectively, and three methods were selected, including Rb, and the $\mathrm{DM}$ algorithm is used in this study. 10 experiments were conducted for each method and were selected for each experiment and analyzed by using the university students' PCS recognition. Error recognition rate and rejection rate are shown in Figures 4-6, respectively.

The correct rate, misidentification rate, and rejection rate of university students' PCS recognition by support vector machine are 85.12 percent, 8.35 percent, and 7.8 percent, respectively, as shown in Figures 4-6. The recognition effect of university students' PCS is not satisfactory. This is due to the high amount of noise in university students' PCS signals. This method ignores noise and, to some extent, interferes with feature extraction, increasing the error rate of university students' PCS recognition. The correct rate, misidentification rate, and recognition effect of university students' PCS are not the best, with 84.67 percent, 8.27 percent, and 6.56 percent, respectively. This is because, despite the fact that this method processes the signal of a university student's PCS with noise, it removes the noise interference. Teachers can use the measurement result management module to filter, query, sort, edit, print, and delete data. The correct rate, misidentification DM algorithm, and correct rate, respectively, are 94.62 percent, 3.45 percent, and 2.53 percent. The correct rate of the DM algorithm is increased by 10.39 percent and 9.65 percent, respectively, when compared to the correct rate of the DM algorithm. To ensure operational efficiency, clear division of labor, clear objectives, and complete functions, the team must continue to promote the development of an intervention system. The information system must also be standardized. There are currently no laws or regulations governing the collection, storage, mining, or use of big data technology. Data collection and use are harmed to some extent, and no clear workflow and requirements for implementation have been established. This is because this method first preprocesses university students' PCS signals and then extracts better recognition DM technology to fit the changing characteristics of university students' PCS, resulting in a better university students' PCS recognition model, reducing university students' PCS recognition based on neural network, and verifying the superiority of the DM algorithm.

In the traditional mode of psychological crisis, researchers collect data of university students' mental health status through clinical scale, psychological investigation, daily outpatient service, etc., and the results are often not shared with counselors, class teachers, and other student workers. It mainly includes the training time and test of university students' PCS identification. In order to reflect the

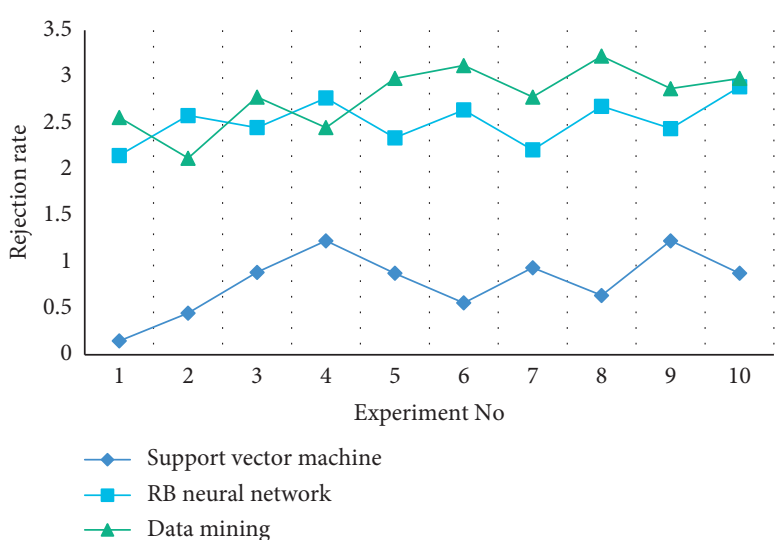

FIGURE 4: Rejection rate of university students' psychological crises by different methods (Experiment 1).

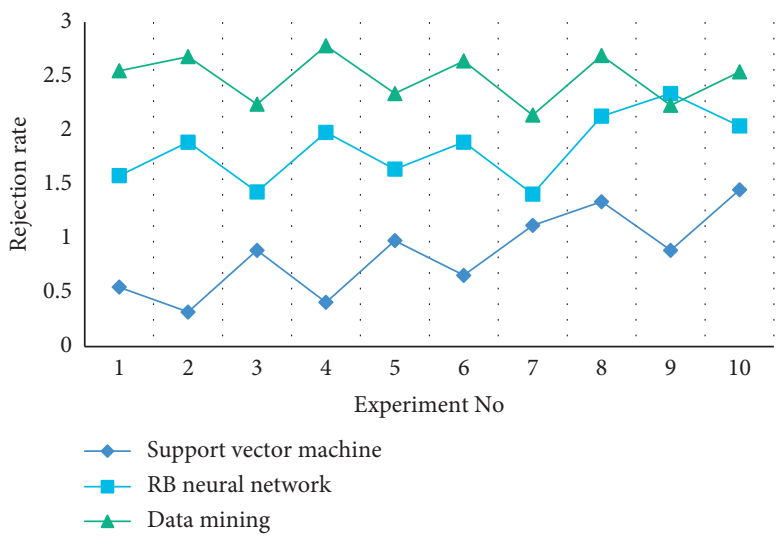

Figure 5: Rejection rate of university students' psychological crises by different methods (Experiment 2).

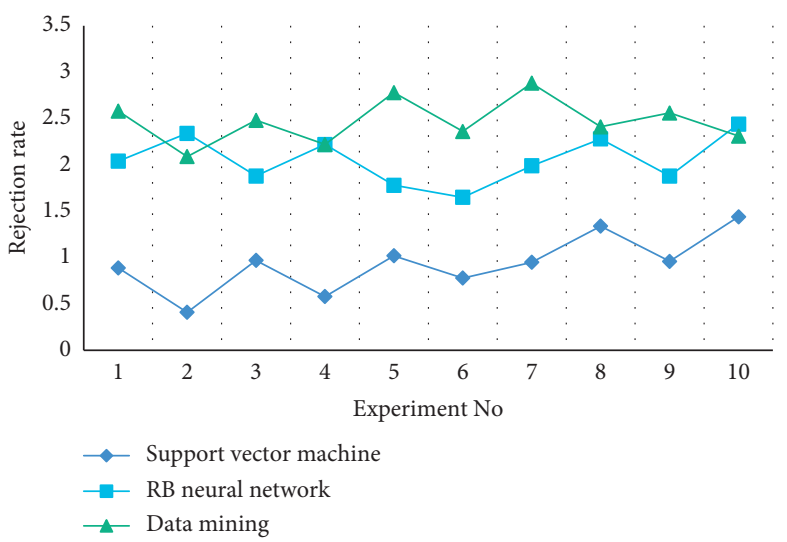

FIGURE 6: Rejection rate of university students' psychological crises by different methods (Experiment 3).

fairness of the experimental results of university students' PCS identification, three methods were used to carry out four experiments for comparison, and each method used was selected for each experiment for simulation test. The sample data distribution university students' PCS identification is shown in Figures 7-10. 


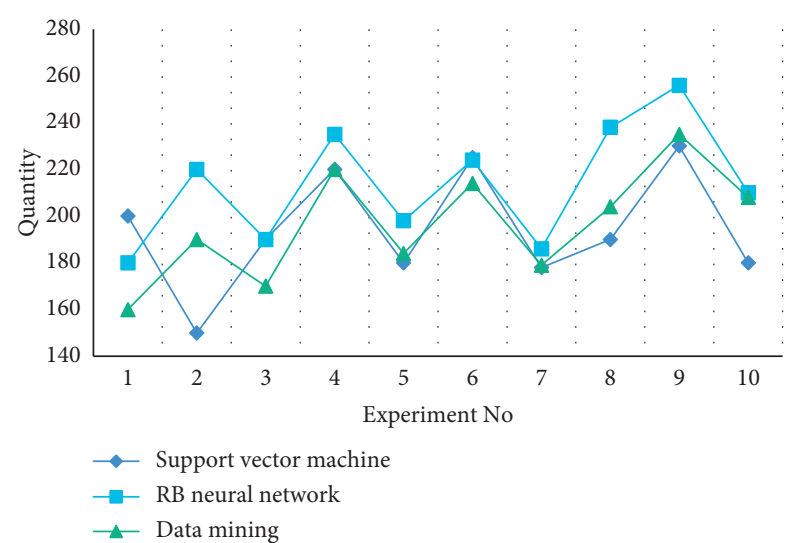

FIgURE 7: Distribution of sample data in the simulation experiment of university students' PCS identification (Experiment 1).

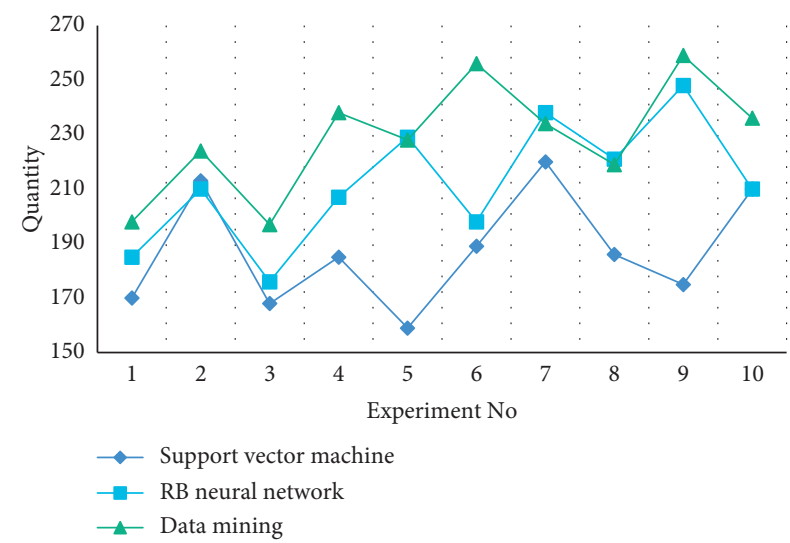

FIGURE 8: Distribution of sample data in the simulation experiment of university students' PCS identification (Experiment 2).

The experimental results from Figure 7 to Figure 10 show that the average modeling time of university students' psychological crisis identification based on the DM algorithm is $18.03 \mathrm{~s}$, the network is $86.5 \mathrm{~s}$, and $38.47 \mathrm{~s}$, respectively. Psychological scale is widely used as a standardized work to evaluate the mental health level of university students, primarily for the investigation and research of university students' mental health. The general survey of newly enrolled students' mental health aims to identify students with psychological problems and assess the mental health of university students. The scale, as a test of students' psychological states, has a direct impact on students, and the scale's compilers are educators who use the scale to form relationships with students. Data on university students are primarily gathered in various school departments. Relevant leaders should pay enough attention to the cooperation between different departments and the data application management system in order to effectively retrieve, apply, and mine data in different departments. Schools should form a big data collection system working group that includes the security department, the school hospital, school leaders, the student affairs office, and the mental health department. Although the scale weavers considered students' characteristics to some extent in the past, they still regarded

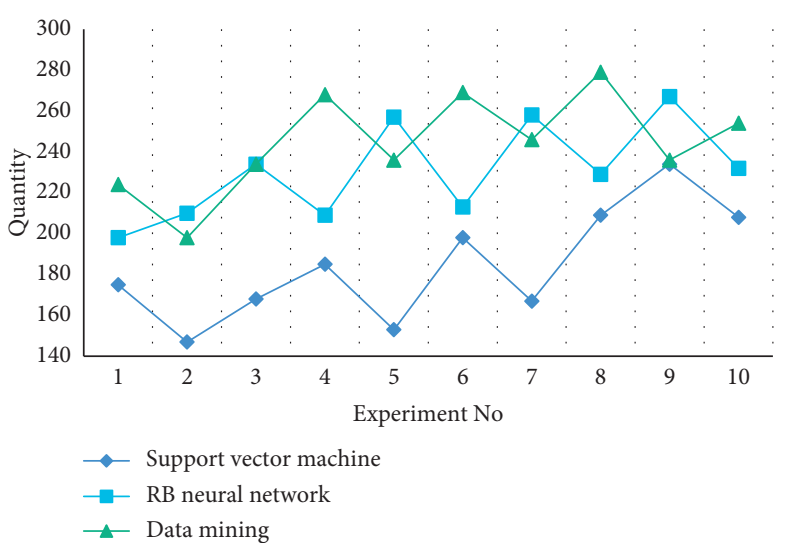

FIgURE 9: Distribution of sample data in the simulation experiment of university students' PCS identification (Experiment 3).

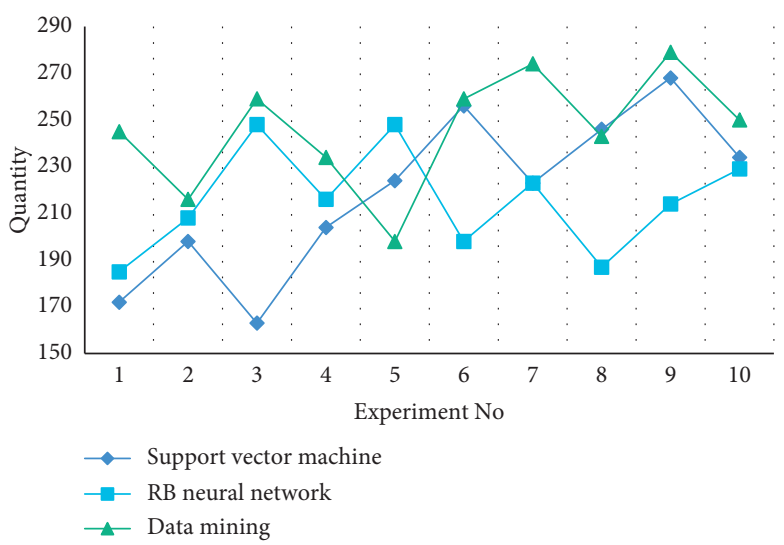

FIGURE 10: Sample data distribution diagram of university students' PCS identification simulation experiment.

students as objects that could be used and transformed, and their inner world could be tested according to the contents of the test scale, influenced by the traditional educational concept. In reality, students' inner worlds are complex, and everyone receives different guidance information from the outside world. This has an impact on the scale's test results, and it makes it difficult to predict and screen out more problematic students. As a result, the DM algorithm reduces the modeling time for university student PCS identification, increases the speed of PCS identification for university students, and has a higher practical application value.

\section{Conclusions}

College students who have self-respect are more likely to accept themselves, accept their own flaws, and appreciate their own advantages, rather than feeling inferior and suppressing themselves because of their own flaws. This paper proposes a method of college students' psychological crisis identification based on data mining technology in order to achieve ideal results in psychological crisis identification. We can combine big data and optimize it through strategies such as building data feedback platform, optimizing management system, and building data collection 
system, in light of the shortcomings of the traditional intervention system, such as full sharing of data mining algorithms, insufficient dynamic management of data information, and inability to fully mine data. The simulation results of college students' psychological crisis state identification show that this method is a high-accuracy, shortmodeling-time method of identifying college students' psychological crisis states, and the identification results can provide important information for college students' psychological managers. The information is kept in a database called the psychological census. Psychological work assesses students' mental health by examining psychological test data from college students' psychological crisis state identification in the screening data, which contains the original student data.

\section{Data Availability}

The data used to support the findings of this study are included within the article.

\section{Conflicts of Interest}

The author declares no conflicts of interest.

\section{References}

[1] M. Dempsey and A. M. Valenti, "Student uklwds," The Journal of Academic Librarianship, vol. 42, no. 3, pp. 200-206, 2016.

[2] X. Wang, "Course-Taking patterns of community college students beginning in STEM: using data mining techniques to reveal viable STEM transfer pathways," Research in Higher Education, vol. 57, no. 5, pp. 544-569, 2016.

[3] P. White, "Using data mining for citation analysis," College \& Research Libraries, vol. 80, no. 1, pp. 76-93, 2019.

[4] A. C. Kelly and E. Stephen, "A daily diary study of selfcompassion, body image, and eating behavior in female college students," Body Image, vol. 17, no. jun, pp. 152-160, 2016.

[5] H. Y. Cheung, T. Teo, and M. T. Hue, "Modeling the relationships among emotional intelligence, sensation-seeking and risk-taking attitudes of university students in Hong Kong [J]," Journal of Risk Research, vol. 20, no. 5-6, pp. 569-589, 2017.

[6] H. Liu, S. He, H. Long et al., "Knowledge, attitudes and practices related to WG consumption among college students: a cross-sectional study in Chongqing, China," Public Health, vol. 190, pp. 37-41, 2021.

[7] M. Li, G. Zhou, W. Cai et al., "Multi-scale sparse network with cross-attention mechanism for image-based butterflies finegrained classification," Applied Soft Computing, vol. 117, Article ID 108419, 2022.

[8] R. Liu, W. Cai, G. Li, X. Ning, and Y. Jiang, "Hybrid dilated convolution guided feature filtering and enhancement strategy for hyperspectral image classification," IEEE Geoscience and Remote Sensing Letters, vol. 19, pp. 1-5, Article ID 5508105, 2022.

[9] J. Kong, C. Yang, J. Wang et al., "Deep-stacking network approach by multisource data mining for hazardous risk identification in IoT-based intelligent food management systems," Computational Intelligence and Neuroscience, vol. 10, 2021.

[10] V. Vaishnavi and M. Suresh, "Assessment of healthcare organizational readiness for change: a fuzzy logic approach," Journal of King Saud University-Engineering Sciences, 2020, In press.

[11] S. Liu, J. Keeley, and W. Buskist, "Chinese college students' perceptions of excellent teachers across three disciplines," Teaching of Psychology, vol. 43, no. 1, pp. 70-74, 2016.

[12] E. Asici and H. I. Sari, "A proposed model to explain happiness in university student: the roles of perceived parenting styles, emotional self-efficacy, andForgiveness[J]," Journal of Adult Development, vol. 28, pp. 1-14, 2021.

[13] X. Zhu and Y. Geng, "Childhood environment, sense of control and hoarding among Chinese university student[J]," Current Psychology, vol. 2021, no. 3, pp. 1-8, 2021.

[14] J. S. Wrench, N. M. PunYaNunt-Carter, and A. J. Garcia, ''Understanding college students' perceptions regarding mindfulness: the impact on intellectual humility, faith development, religious communication apprehension, and religious communication," Journal of Religion and Health, vol. 59, no. 4, pp. 1794-1809, 2020.

[15] K. Morehead, J. Dunlosky, K. A. Rawson, R. Blasiman, and R. B. Hollis, "Note-taking habits of 21st Century university student: implications for student learning, memory, and achievement[J]," Memory, vol. 27, no. 5-6, pp. 807-819, 2019.

[16] B. Borsari, M. M. Yalch, P. Pedrelli, S. Radomski, R. L. Bachrach, and J. P. Read, "Associations among trauma, depression, and alcohol use profiles and treatment motivation and engagement in university student[J]," Journal of American College Health, vol. 66, pp. 1-25, 2018.

[17] B. Güler, M. L. Cherry, M. A. Cherry, S. Aarstad-Martin, C. Cloud, and L. M. Shamp, "Posttraumatic stress, coping flexibility, and risky drinking among trauma-exposed male and female university student: the mediating effect of delay of gratification[J]," Substance Use \& Misuse, vol. 53, no. 3, pp. 508-520, 2018.

[18] Z. Drezner, J. Brimberg, N. Mladenovic, and S. Salhi, "New local searches for solving the multi-source Weber problem [J]," Annals of Operations Research, vol. 246, no. 1-2, pp. 181-203, 2016.

[19] D. E. Taylor, "College students and nature: differing thoughts of fear, danger, disconnection, and loathing," Environmental Management, vol. 64, no. 1, pp. 79-96, 2019.

[20] S. L. West, C. W. Graham, and P. Temple, "Rates and correlates of binge drinking among university student with disabilities, United States, 2013[J]," Public Health Reports, vol. 132, no. 2, pp. 496-504, 2017.

[21] S. Nallasivan, "AB1071How much is the awareness about rheumatology? Survey among medical students (yr 3) in velammal medical college, madurai, tamilnadu, India[J]," Annals of the Rheumatic Diseases, vol. 75, no. Suppl 2, p. 1268, 2016.

[22] K. A. Tyler, R. M. Schmitz, C. M. Ray, S. A. Adams, and L. G. Simons, "The role of protective behavioral strategies, social environment, and housing type on heavy drinking among university student[J]," Substance Use \& Misuse, vol. 53, pp. 1-10, 2017.

[23] B. Andraka-Christou, B. Alex, and J. L. Madeira, "College student preferences for substance use disorder educational videos: a qualitative study[J]," Substance Use \& Misuse, vol. 54, pp. 1-8, 2019. 
[24] M. B. Benz, A. M. Dibello, S. G. Balestrieri et al., "Off-campus residence as a risk factor for heavy drinking among university student[J]," Substance Use \& Misuse, vol. 52, pp. 1-6, 2017.

[25] T. Lorberbaum, K. J. Sampson, J. B. Chang et al., "Coupling data mining and laboratory experiments to discover drug interactions causing QT prolongation," Journal of the American College of Cardiology, vol. 68, no. 16, pp. 1756-1764, 2016. 